\title{
Method for an electronic controlled platooning system of agricultural vehicles
}

\author{
X. Zhang, Prof. Dr. M. Geimer, L. Grandl and B. Kammerbauer
}

\begin{abstract}
This paper presents a method to develop an electronic controlled platooning system, which will enable an autonomous agricultural vehicle to follow a leading tractor with a given lateral and longitudinal offset. In our study not only the follow-up motions but also the problems such as avoiding obstacle, turning at the end of the field have been considered. With the aid of the RTK GPS systems the position of the leading tractor can be obtained with accuracy in the range of centimeters. The position points of the leading tractor are transmitted by wireless modems to the following vehicle continually to provide the target position points for the guidance of the following agricultural vehicle. With the method of curve fitting a desired path for the following vehicle could be dynamically created. Based on the target position points and the path planning, the desired speed and the desired steering angle of the following tractor are calculated. In order to ensure the precise navigation of the driverless following tractor, a course tracking controller and a speed controller have to be designed and implemented. In addition to the motion control algorithms which could keep the autonomous agricultural vehicle following the leading tractor, considerations about safety and robustness of the whole platooning control system would be issued at the end of this paper.
\end{abstract}

Index Terms - Autonomous agricultural vehicle; Navigation and control; Vehicular Safety; Inter-Vehicle-Communication

\section{INTRODUCTION}

The demand on increasing agricultural productivity and the decreasing labor force in the agricultural industry have made the automation of agricultural machinery inevitable. Automation and electrification in the agricultural machinery have become a trend in the recent years. With the modern technology such as real-time kinematic (RTK) GPS systems the accuracy of the positioning can reach 1 to $2 \mathrm{~cm}$ per 10 $\mathrm{km}$ [1], which makes the precise navigation of agricultural machinery possible. With the introduction of X-BY-Wire technology in the modern agricultural machinery the automatic guidance of land vehicles has become reality and more and more functions in agricultural vehicles have be

This work is supported by the Federal Ministry of Food, Agriculture and Consumer Protection of the German Government.

$\mathrm{X}$. Zhang is with the Institute for Vehicle Science and Mobile Machines at the Karlsruhe Institute of Technology (KIT), Gotthard-Franz-Str. 8, D76131 Germany. (phone: 0049-721-6088640; fax: 0049-721-6088609; email: xi.zhang@ @it.edu).

Prof. Dr. M. Geimer is head of the Institute for Vehicle Science and Mobile Machines at KIT. (e-mail: geimer@kit.edu).

L. Grandl is with the AGCO Corporation at Marktoberdorf, Germany. (e-mail: Ludwig.grandl@xfendt.de).

B. Kammerbauer is with the geo-konzept $\mathrm{GmbH}$ at Adelschlag, Germany. (e-mail: bkammerbauer@geo-konzept.de). automated using electronic control systems [2]. In the past ten years, many research works have been carried out to develop an automated agricultural vehicle to replace the labor workforce in the farming operation. In [3] an automatic steering system was developed to guide a John Deere 7800 tractor along prescribed straight row courses with an average error of approximately $2 \mathrm{~cm}$. In [4] a robot tractor was developed based on RTK-GPS and gyroscope to provide navigation information for the path tracking. Such field robot with auto-steering systems are capable of steering along target lines automatically, but the application of such autonomous agricultural vehicles can only be confined to a laboratory environment, where obstacles and other safety related problems could be foreseen. To solve the safety problems in the real field operations many other high-tech sensors have been used to sense the surrounding environment of the farming vehicles. In [5] a machine vision based guidance system was demonstrated for an autonomous agricultural small-grain harvester using a cab-mounted camera. In the recent years laser or laser radar (ladar) have been more and more applied in autonomous vehicles to detect obstacles for the safety reasons. In [6] ladar has been used to navigate a small robot tractor through an orchard field. However most of the solutions have been successfully realized only in laboratory conditions. Field trials demonstrated that an automatic guided agricultural vehicle could assist the operator but could not completely replace the operator because of safety considerations. Some solutions which have been proved robust in field tests were very costly and still a long way from commercialization. On such a background an electronic controlled platooning system can be regarded as an intermediate step on the road to completely autonomous agricultural vehicles. Because of the presence of the operator on one of the agricultural vehicles, the safety problem can be easily resolved without consideration of costly sensors and complicated sensor fusion algorithm. Thanks to the auto-steering and auto-cruise control, which have been implemented as standard technique in commercial agricultural vehicles, the operator can be relieved from the tedious driving routines and concentrate himself mainly on the supervising of the working processes. The primary objective of this paper is to introduce a method to develop an electronic controlled two-tractor platooning system, which will enable one unmanned tractor to follow up another leading tractor with a given lateral and longitudinal offset. This system can allow one operator to utilize more than two agricultural machines simultaneously, so that the productivity of the working process will be substantially improved and the competitiveness of the agriculture 
producer will be enhanced. In addition to the follow-up motion which has been demonstrated in many other research works, the obstacle avoidance maneuver and the turn-around of the vehicle at the end of a row will be considered. The safety and robustness of such a platooning system will be issued as well.

\section{EXPERIMENTAL EQUIPMENTS AND METHODS}

Fig. 1 shows one of the experimental agricultural vehicles, which are used to compose the platooning system. The leading vehicle as well as the following vehicle is a $265 \mathrm{~kW}$ four-wheel drive Fendt 936 Vario model which is $5.65 \mathrm{~m}$ long, $2.75 \mathrm{~m}$ wide and $3.37 \mathrm{~m}$ high. The equipment used to measure the tractor position of the leading tractor is different from the following tractor. The leading tractor uses a Trimble navigation system, which was mounted by the geokonzept GmbH. With the AgGPS 252 GPS-receiver attached to the roof of the cab and the 450 radio equipment which receives the real-time kinematic (RTK) signals at $10 \mathrm{~Hz}$ data throughput rate, the position accuracy is less then $2.5 \mathrm{~cm}$. Using data from the GPS receiver and internal sensors the position data can be further corrected by the NavController in the cab which can compensate the roll, pitch and yaw movement of the vehicle during measurement.

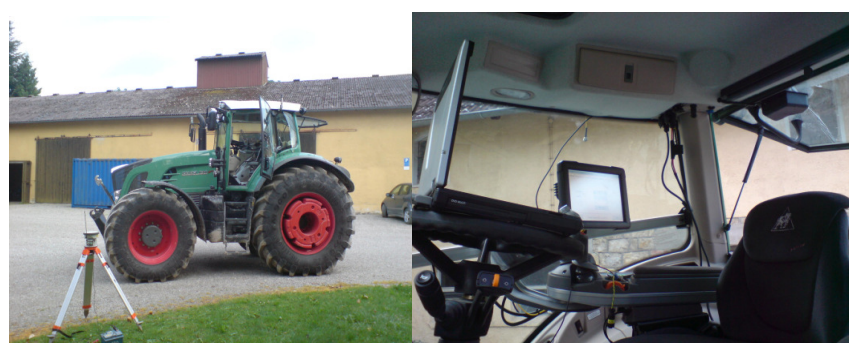

Fig. 1 Fendt 936 Vario tractor and its cabin's inner view

In the following tractor an auto-guide system was already installed to measure the position of the vehicle. This system is an accessory equipment of the Fendt 936 Vario tractor and can correct the positioning error caused by the inclination of the ground. A gyroscope is also integrated in this auto-guide system, so that the positioning can reach the same accuracy as the Trimble system. Both tractors are equipped with an industrial computer, an AutoBox which is the interface between the GPS receiver and the tractor control unit (Fig. 2). The industrial computer AutoBox is composed of a PowerPC 750GX processor board running at $1 \mathrm{GHz}$ and several peripheral boards, which can communicate with external equipments over controller area network (CAN) or serial interfaces. With the real-time operating system running on the PowerPC the AutoBox performs data collection, condition monitoring and control signal computations using software written at KIT.

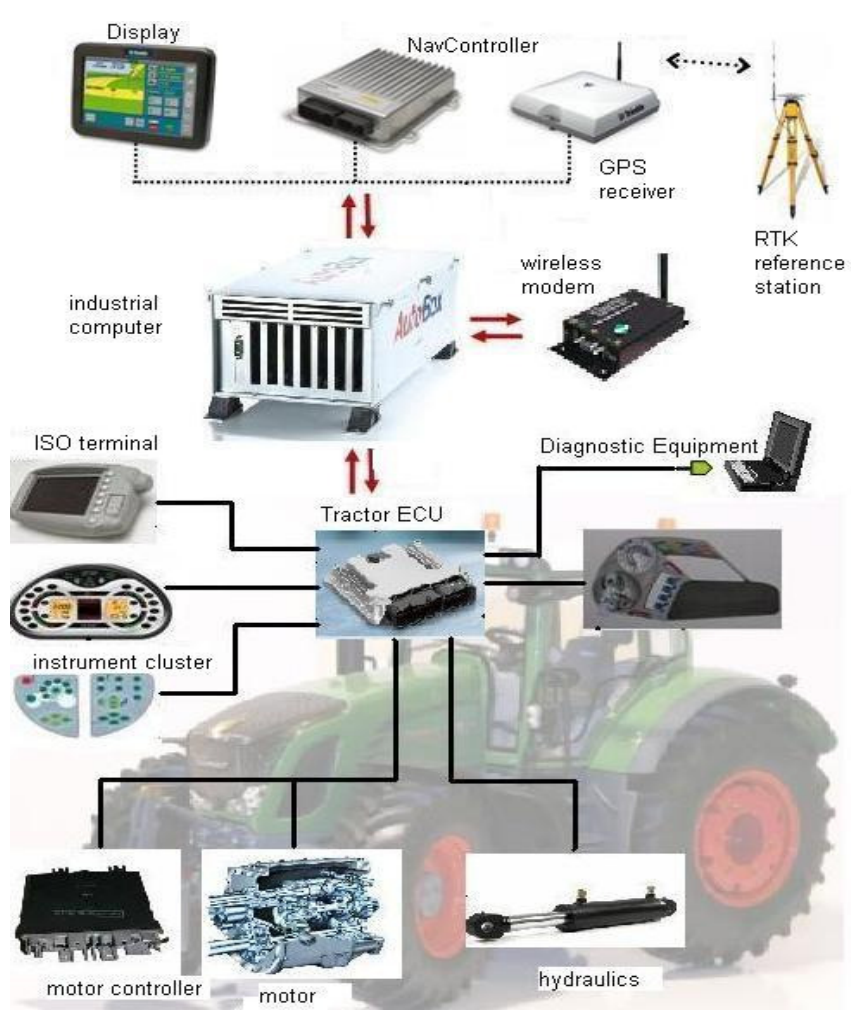

Fig. 2 Hardware architecture of the tractor in the platooning system

In Fig. 3 a method to design a platooning system for two tractors is demonstrated. The leading tractor is driven by an operator and collects its position, heading angle and velocity every $100 \mathrm{~ms}$ from satellites. Its position will be corrected by the RTK signal from a reference station and by the navigation controller in the cab. The corrected kinematic information will be read into the AutoBox over a CAN interface. Other status information about the leading tractor will also be collected either periodically or on demand by the AutoBox from the CAN interface. All these information is shaped in desired data set and will be transferred to the following tractor according to priorities. Once the current position of the leading tractor is obtained, the trajectory of the leading tractor will be updated using curve fitting. According to the updated trajectory of the leading tractor, the new path segment for the guidance of the following vehicle can also be obtained every $100 \mathrm{~ms}$.

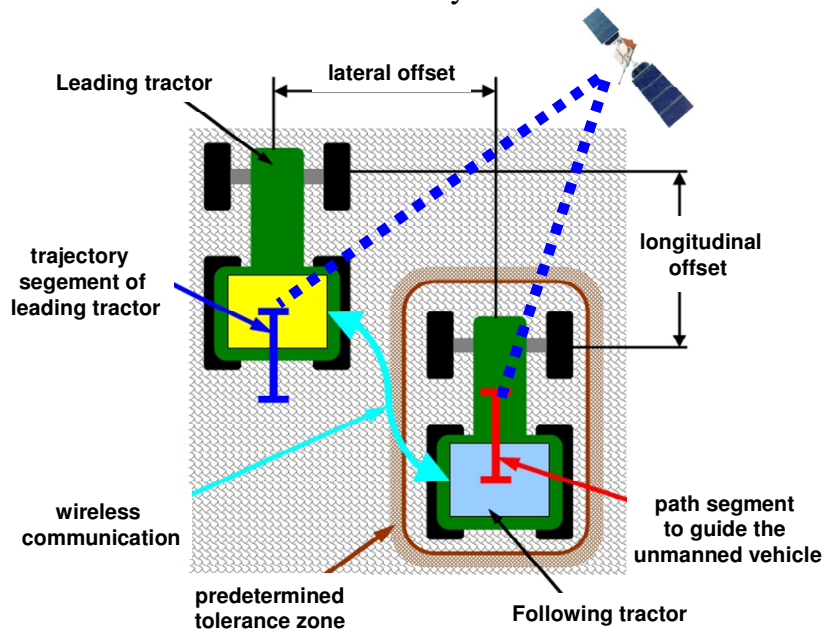

Fig. 3 Schematic diagram of the platooning system for two tractors using GPS and wireless communication. 
A vital part of an autonomous, unmanned vehicle is safety. In such a platooning system, the presence of the operator enhances the safety of the system in unexpected dangerous situations. To disburden the operator from the routine supervising work and assist him by decision making, programs doing condition monitoring have been integrated in the software. One of the most important condition monitoring for a platooning system is the distance monitoring. A virtual rectangle safety zone, which always surrounds the unmanned following tractor during its moving, is conceived to constrain the movement of the tractor and to prevent it from colliding against the leading vehicle. When the following tractor goes beyond the constraints determined by this safety zone, it will be halted by a real-time program, which will steadily monitor the position of the unmanned vehicle. In order to prevent the unmanned tractor from being frequently halted, a predetermined tolerance zone is considered. When the tractor exceeds the inner edge of this tolerance zone, a warning signal will be created to notify the operator on the leading tractor that the following tractor is already in the tolerance zone and a suggestion will be given according to whether the following tractor is moving too slow or too fast. For example, if the following tractor has a heavier implement than the leading tractor, it needs more power to overcome the greater tractive resistance, because its implement is deeper in the soil. When the following tractor reaches its power limit, its velocity will be decreased so that it could probably not follow up the leading vehicle. In such a situation the operator on the leading vehicle will obtain a suggestion to slow down its velocity, so that the following vehicle can still keep up with the leading one. When the following vehicle lags more behind in spite of the decreased driving speed of the leading tractor and exceeds the extern edge of the tolerance zone, both tractors will be halted immediately and a manual inspection must be done. The width and length the safety zone and the thickness of the tolerance zone can all be tuned by the operator before the system is coupled for platooning.

\section{PATH PLANNING}

\section{A. Follow-Up}

The most commonly used motion mode for a platooning system is the follow-up motion [7]. Fig. 4 shows an example how the desired path for the following vehicle is created from the trajectory of the leading tractor in the follow-up mode.

The solid curve on the left side shows the trajectory of the leading tractor $\boldsymbol{c}_{\text {master }}$. The current position of the leading tractor $\left[\mathrm{x}_{\mathrm{k}}, \mathrm{y}_{\mathrm{k}}, \psi_{\mathrm{k}}\right]$ is obtained in equally spaced point in time $(\Delta \mathrm{t}=100 \mathrm{~ms})$ from the navigation controller and its mapping point on the desired path for the following vehicle $\left[\mathrm{x}_{\mathrm{k}}^{\prime}, \mathrm{y}_{\mathrm{k}}^{\prime}, \psi_{\mathrm{k}}^{\prime}\right]$ will be calculated with a given lateral offset $d$ as:

$$
\begin{aligned}
& x_{k}{ }^{\prime}=x_{k}+d \sin \psi_{k} \\
& y_{k}{ }^{\prime}=y_{k}-d \cos \psi_{k} \\
& \psi_{k}{ }^{\prime}=\psi_{k}
\end{aligned}
$$

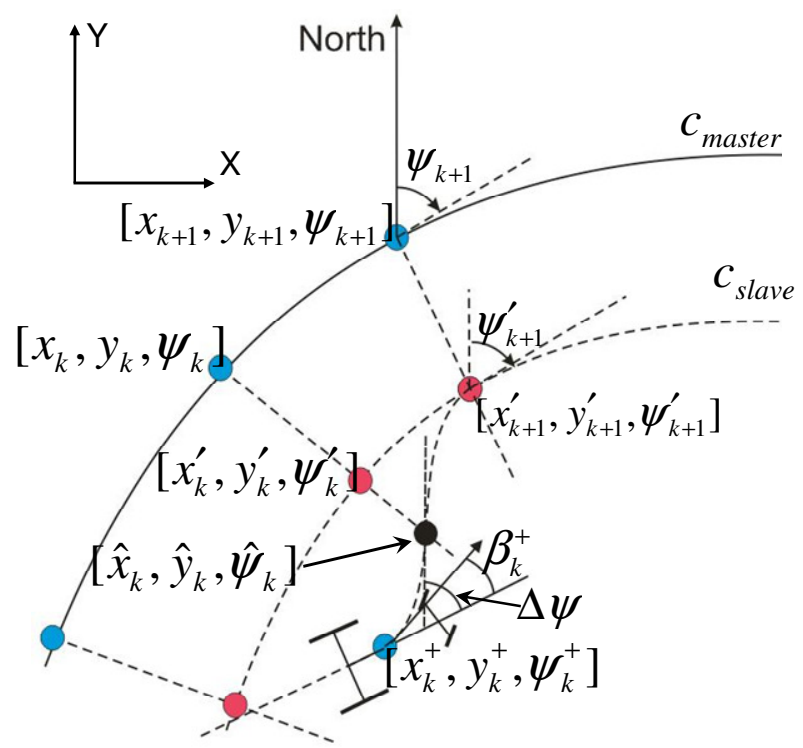

$$
\begin{array}{rr}
{\left[x_{k}, y_{k}, \psi_{k}\right] \text { :current posture of }} & {\left[\hat{x}_{k}, \hat{y}_{k}, \hat{\psi}_{k}\right] \text { :target point on }} \\
\text { the leading tractor } & \text { the desired path } \\
{\left[x_{k}^{\prime}, y_{k}^{\prime}, \psi_{k}^{\prime}\right] \text { :mapping point on }} & {\left[x_{k}^{+}, y_{k}^{+}, \psi_{k}^{+}\right] \text {: current posture of }} \\
\text { the desired path } & \text { the following tractor }
\end{array}
$$

Fig. 4 Path planning for the following vehicle in the follow-up mode.

That means the mapping point locates always in the direction which is perpendicular to the heading of the leading tractor.

Together with the previous mapping points, the desired path $\boldsymbol{c}_{\text {slave }}$ for the following vehicle can be updated using curve fitting every $100 \mathrm{~ms}$. The instantaneous target point for the navigation of the following vehicle $\left[\hat{x}_{k}, \hat{y}_{k}, \hat{z}_{k}\right]$ will be selected on a path segment, which is between its current position $\left[x_{k}^{+}, y_{k}^{+}, z_{k}^{+}\right]$and its desired position on $\boldsymbol{c}_{\text {slave }}$ for the next step $\left[x_{k+1}^{\prime}, y_{k+1}^{\prime}, z_{k+1}^{\prime}\right]$. This path segment between the current position of the following vehicle and its desired position for the next step can be shaped using a spline function as:

$$
y(x)=a_{0}+a_{1} \cdot x+a_{2} \cdot x^{2}+a_{3} \cdot x^{3}
$$

With the boundary conditions of gradients as:

$$
\begin{aligned}
& y_{k+1}^{\prime}\left(x_{k+1}\right)=1 / \tan \psi_{k+1}^{\prime} \\
& y_{k}^{\prime}\left(x_{k}\right)=1 / \tan \left(\psi_{k}^{+}-\beta_{k}^{+}\right)
\end{aligned}
$$

The target point is obtained as the intersection of this spline and the normal of the curve $\boldsymbol{c}_{\text {slave }}$ at the point $\left[x_{k}^{\prime}, y_{k}^{\prime}, z_{k}^{\prime}\right]$.

\section{B. Obstacle Avoidance}

The most difficult and perhaps the most crucial issue related to the safety of an unmanned vehicle is the obstacle avoidance. This problem has been investigated by many researchers [8] [9] [10] [11], but few have provided absolutely convincing and reliable solutions to prevent the autonomous vehicle from colliding against the obstacle in unforeseeable circumstances. Therefore we have chosen the operator to detect an obstacle which may lay on the desired path of the following vehicle. When the operator perceives a 
collision risk for the following vehicle, he can guide the unmanned vehicle to avoid the obstacle by starting a "LaneChange" maneuver, just with a button press. Fig. 5 shows how the following vehicle can take a path with continuous curvature to follow the trajectory of the leading one, when the operator on the leading vehicle sends a "Lane-Change" command and return to its previous lane, when it has avoided the obstacle. In the "Lane-Change" maneuver the normally used third order curve fitting is not sufficient to provide a smooth transition. According to [12], a fifth degree polynomial:

$$
y(x)=a_{0}+a_{1} \cdot x+a_{2} \cdot x^{2}+a_{3} \cdot x^{3}+a_{4} \cdot x^{4}+a_{5} \cdot x^{5}
$$

delivers a smooth change between the two transition points (1) and (2).

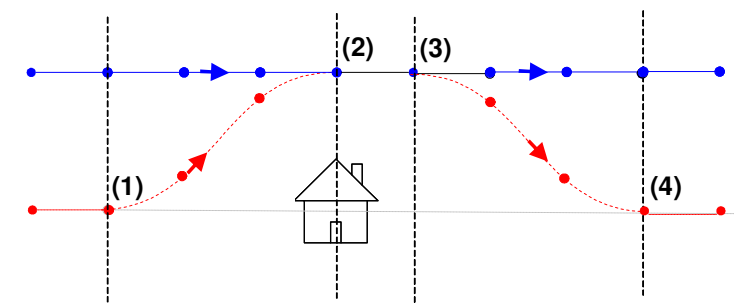

Fig. 5 Path planning for a "Lane-Change" maneuver to avoid the collision

After the operator ensures that the following vehicle has already avoided the obstacle, he will send a signal to cancel the "Lane-Change", so that the following vehicle can calculate its path to return to its previous lane with a given lateral offset.

\section{Turn-Around}

Fig. 6 shows two most common patterns for the unmanned following tractor to turn around [13] [14], when it reaches the field end. On the left the example solution for a left hand side turning is presented.

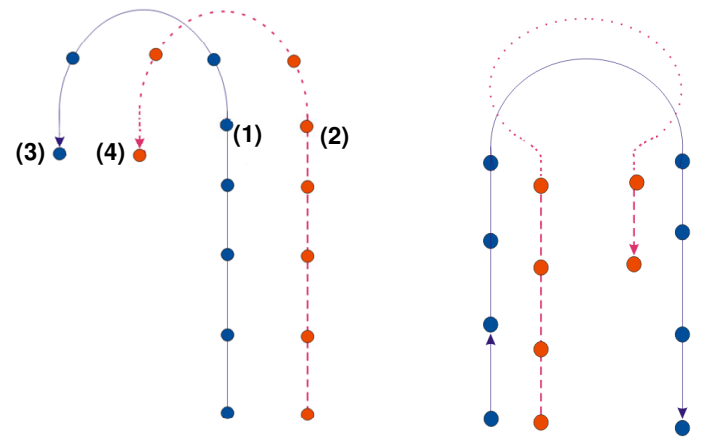

Fig. 6 Path planning for the following tractor by turning

At the point (1) the operator starts the turning of the leading tractor. The following tractor must halt at the mapping point (2) of the point (1) and wait until the operator has finished the turning manually and reached the point (3). Then the following vehicle can start its turning autonomously along the pre-calculated turning path through the mapping points (2) and (4). After the following tractor has reached the point (4) the whole turning process is completed. A remarkable feature of this turning strategy is that the following tractor changes its relative position to the leading vehicle from the right side before the turning to the left side after the turning. This side change must be taken into account before the platooning system starts the followup motion again.

Another turning maneuver is presented in Fig. 6 on the right side, the so-called loop turn [15]. In this turning maneuver the following tractor remains at the right side of the leading vehicle, so that the side change of the following tractor doesn't come into question.

\section{CONTROLler DESIGN}

A control structure which contains cascade controller with feed forward control is designed to guide the unmanned tractor along the calculated desired path and to minimize the path error [16]. Fig. 7 demonstrates the structure for the speed control which will adjust the velocity of the following vehicle to keep its distance from the leading tractor constant.

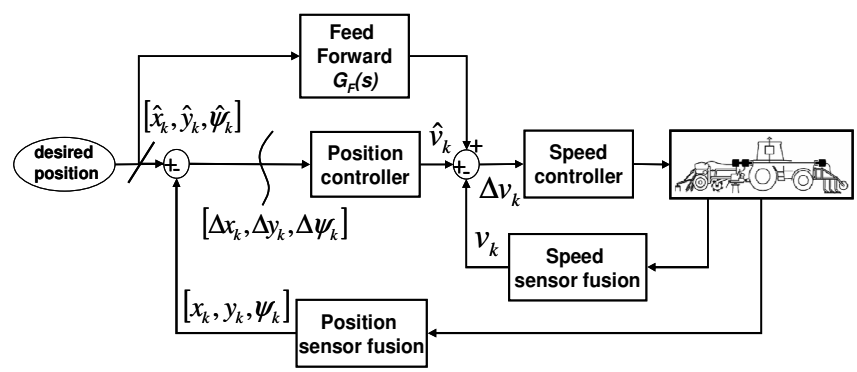

Fig. 7 Structure of the cascade controller with feed-forward control for the following vehicle speed.

In this control structure the current position and speed of the following vehicle are obtained using the sensor fusion algorithm which was programmed in the navigation controller. The position and the heading angle of the following vehicle will be compared with the calculated desired position and the desired heading angle from the path planning. Using the feed-forward function $G_{F}(s)$ a calculated bias will be added to the output from the position controller. The sum of both signals will then be compared with the current speed of the following vehicle obtained from sensor fusion. The output of the speed controller is connected to a CAN interface and can drive the tractor electronic control unit (ECU) to control the mechanical components in the agricultural machine.

The structure for the steering angle control is similar to the structure explained above. In this case the position controller will be replaced by a yaw-angle controller, while the speed controller will be replaced by a steering angle controller.

\section{INTER-VEHICLE COMMUNICATION}

\section{A. Hardware}

One of the most important prerequisites for an electronic controlled platooning system is that the leading and the following vehicles are connected by a so-called wireless CAN-bridge, which can collect the data from the controller area network (CAN) bus in one vehicle, transmit it over the air and send the information again to the CAN bus in the other vehicle. Because of the normally large acreage of a farm, a wide-coverage mobile communication device with real-time link ability must be chosen to satisfy the 
requirements for such an inter-vehicle communication [17]. The wireless connection has been successfully established using two modems working on the $2.4 \mathrm{GHz}$ ISM band and on the $869 \mathrm{MHz}$ band respectively. The AutoBox, which is connected with the wireless modems by serial interfaces (RS-232) and with the vehicle controllers by CAN Interfaces, coordinates the data transmission between CAN bus and the wireless modems. Table 1 shows the specifications of the two wireless modems. Principally we need to transmit two types of information between the vehicles: the status information, which must be transmitted cyclically, and the command information, which is transmitted once only when some operation is to be carried out. The $2.4 \mathrm{GHz}$ modem is appropriate for the transmission of cyclic information because of its larger bandwidth and $100 \%$ duty cycle, whereas the $869 \mathrm{MHz}$ modem has a larger long range but lower throughput $(24 \mathrm{kpbs} \times 10 \%)$, which is however sufficient for the transmission of acyclic command information.

\begin{tabular}{|lll|}
\hline \multicolumn{2}{|l|}{ Specification of communication device } \\
& XStream-PKG & XBee-PRO 868 \\
Frequency range & $2.4 \mathrm{GHz}$ & $\mathbf{8 6 9 . 5 2 5 M H z}$ \\
Modulation & FSK & OQPSK \\
Bandwidth & $400 \mathrm{kHz}$ & $\mathbf{2 5 0 \mathrm { kHz }}$ \\
Channel rate & $20 \mathrm{Kbps}$ & $\mathbf{2 4 k b p s}$ \\
RF output power & $50 \mathrm{~mW}$ & $\mathbf{1 - 3 1 5} \mathbf{~ m W}$ \\
Max long range & $5 \mathrm{~km}$ & $\mathbf{1 6 k m}$ \\
Duty Cycle & $\mathbf{1 0 0 \%}$ & $\mathbf{1 0 \%}$ \\
\hline
\end{tabular}

Tab.1 Specification of the communication devices

\section{B. Data Protocol}

A data protocol, which defines the data type and frame format for all the information to be transmitted by the wireless modems, has been created to distinguish communication data with different content and different priorities.

\begin{tabular}{|c|c|c|c|c|c|c|c|c|}
\hline Field & Delimiter & Frame-ID & UTC & Longitud & Latitude & Heading & Speed & rection \\
\hline Bytes & & 1 & 4 & 6 & 6 & 2 & 2 & 2 \\
\hline Data & $0 x F F$ & $0 \times 02$ & $x x x$ & $x x x x x x$ & $\mathbf{X X X X X X}$ & $\boldsymbol{X X}$ & $x \mathbf{x}$ & $x x$ \\
\hline \multicolumn{9}{|c|}{$\begin{array}{l}\text { Delimiter: Check byte for the start of the frame } \\
\text { Frame-ID: Identification for the data frame, } 2 \text { stands for the position } \\
\text { UTC: Cota from the leading vehicle to the following one } \\
\text { Longitude:Longitude of the current position of the leading vehicle } \\
\text { Latitude: Latitude of the current position of the leading vehicle } \\
\text { Heading: Angle where the leading vehicle is pointing compared to } \\
\text { the true north } \\
\text { Speed: Velocity of the leading vehicle } \\
\text { Direction: Direction in which the leading vehicle are moving }\end{array}$} \\
\hline
\end{tabular}

Tab. 2 Extraction from the data protocol which defines the type and the frame format of the data transmission

In Table 2 the position data of the leading vehicle is defined in a data frame with 32 bytes and with a frame identifier (frame-ID) of 2. Its frame-ID indicates that this information has a relative higher priority in the whole data list. That reflects apparently the fact that the position data is very crucial for the safety of the following tractor. Without this information the unmanned vehicle could not be guided correctly and there would be collision danger.

\section{SAFETY CONSIDERATIONS}

An emphasis of this work is placed on the safe operation of agricultural vehicles. Using the auto-steering and auto- cruise control system which disburden the operator from the tedious farming routine such as driving and turning, the operation safety of the leading vehicle will be enhanced. Supervision software, which assisted the operator to monitor the driving and working status of the unmanned vehicle, also guaranteed the safety of the following vehicle in normal circumstances. As a backup of the supervising software the operator can trigger the emergency stopping to halt the following vehicle immediately in unexpected dangerous situations.

A key issue concerning the development of an electronic controlled, safety-related system is to determine the safety integrity level needed for all subsystems. Using the risk graph defined in the international standard IEC 61508 [18], the severity level of injury and the required performance levels can be derived when the corresponding subsystem fails. As an example, a risk assessment has been conducted for the wireless communication used in the platooning system (Fig. 8). The break of the wireless communication can cause severe injury (S2) because without the information about the leading vehicle the following one could not be guided correctly and there would be collision danger; the frequency of its exposure to hazard is relative high (F2) because of other interferences in the air; the possibility to avoid the hazard exits by triggering an emergency stopping when the acknowledgement for a successful data transmission cannot be obtained by the sender in a certain time period. Therefore the risk assessment of the wireless communication will take the red path in the risk graph. The result is the safety integrity level of 2 and a fail-silent performance is needed for this level. That means the whole system must be shut down, when this subsystem fails. Using a 10o2D-architecture [18], the safety integrity level of the wireless communication can be enhanced to 3 . That means the whole system can still work in fail-tolerant mode when a redundant wireless modem is used in this architecture.

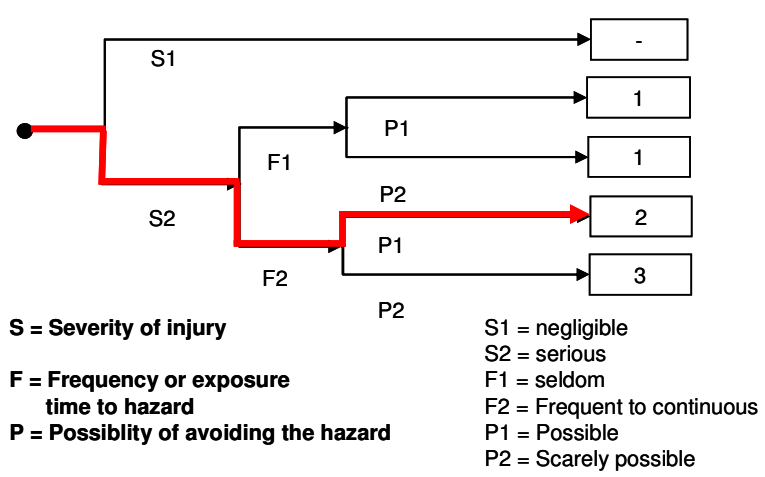

Fig. 8 Classification of safety-related system in different safety-integritylevel according to IEC 61508

According to the different safety-integrity-levels required for each subsystem, the signals to be supervised in the system are classified in three levels: the warning level with emergency stopping, the warning level with fault tolerance and the non-warning information level. A user-friendly interface has been developed by the researchers from the AGCO Corporation to show the operator the warning signals when the system goes into fail-silent or fail-tolerant states (Fig. 9). 


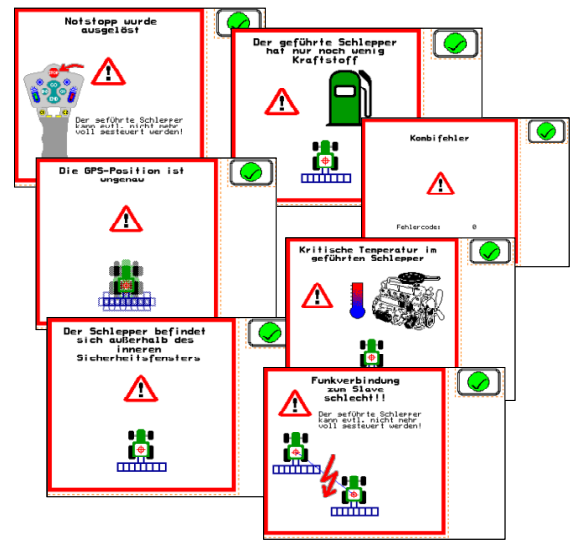

Fig. 9 Screenshots of the User Interface while diagnostic software detects fault condition.

\section{CONCLUSIONS}

In this paper we presented an approach for developing a platooning system, which is able to automate an unmanned agricultural vehicle to fulfill some agricultural task, such as plowing and drilling, cooperatively with another manned leading tractor. Compared with other autonomous agricultural robots which are still far from commercialization, the experimental prototype designed according to our proposal will be able to be converted in a commercialized product in the near future. A lot of considerations have been dedicated to designing a safe and robust system, so that the developed platooning system can meet the common demands regulated in the safety standards for electronic controlled vehicles. An interesting and novel facet of this research is the tolerance zone which constrains the movement of the autonomous vehicle. Significant challenges still lay ahead to determine the dimension of this tolerance zone and to control the unmanned vehicle accurately so that it can always stay in this tolerance zone. Another advantage of our proposal is the supervision of the operator as a safety back-up of the system. Preliminary results from our computer simulation have shown that the following vehicle can follow the leading one satisfactorily.

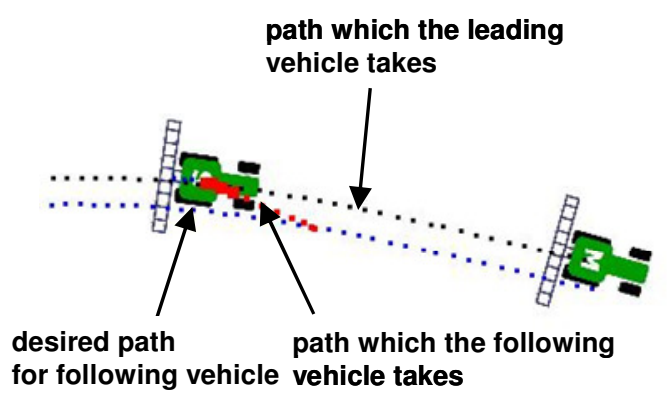

Fig. 10 Simulation of lane change using the methods described in the path planning.

\section{REFERENCES}

[1] T. Muhr, P.O. Noack "Mobile Data Repeaters Enhancing the Availability of RTK Correction Data in the Field" in Proc. of the ATOE Conference, Sep. 2006, Bonn, Germany, pp.65-69.

[2] M. Ehrl, H. Auernhammer " $X-B y$-Wire via ISOBUS Communication Network" in Proc. of the ATOE Conference, Sep. 2006, Bonn, Germany, pp.227-236.

[3] M. O'Connor, T. Bell, G. Elkaim, B.W. Parkinson “Automatic steering of farm vehicles using GPS" in Proc. of the 3rd International
Conference on Precision Agriculture, Minneapolis, MN, June 23-26, 1996, pp.767-778.

[4] N. Noguchi, J.F. Reid, Q. Zhang, J.D. Will, K. Ischii, 2001. "Development of robot tractor based on RTK-GPS and gyroscope" ASAE Paper 01-1195.

[5] E.R. Benson, J.F. Reid, Q. Zhang, "Machine vision-based guidance system for agricultural grain harvesters using cut-edge detection" Biosystems Engineering, vol.86, no.4, pp.389-398, 2003.

[6] R. Tsubota, N. Noguchi, A. Mizushima, "Automatic guidance with a laser scanner for a robot tractor in an orchard" in Proceedings of the Automation Technology for Off-Road Equipment Conference, Kyoto, Japan, 2004.

[7] Z. Zhu, J. Takeda, R. Torisu, J. Chen, Z. Song and E. Mao, "Control System for Tractor-Platooning" in Proceedings of the IEEE International Conference on Mechatronics and Automation, August 5-8, 2007, Harbin, China.

[8] A. Stentz, C. Dima, C. Wellington, H. Herman, D. Stager, "A System for Semi-Autonomous Tractor Operations" Autonomous Robots, vol.13, no.1, July 2002, pp. 87-104(18)

[9] N. Nourani, M. Bosse, J. Roberts, and M. Dunbabin, "Practical path planning and obstacle avoidance for autonomous mowing" in Proceedings of the Australasian conference of robotics and automation, 2006.

[10] V. Subramanian, T.F. Burks and A.A. Arroyo, "Development of machine vision and laser radar based autonomous vehicle guidance systems for citrus grove navigation" Computers and Electronics in Agriculture, vol. 53, 2006, pp. 130-143.

[11] V. Subramanian, T.F. Burks, S. Singh, "Autonomous greenhouse sprayer vehicle using machine vision and ladar for steering control" Applied Engineering in Agriculture, vol. 21, no. 5, pp. 935-943.

[12] W. Nelson "Continuous-Curvature Paths for Autonomous Vehicles" in Proceedings of the IEEE International Conference of Robotics and Automation, Scottsdale, AZ, 1989, pp. 1260-1264.

[13] N. Noguchi, J. Will, J. Reid, Q. Zhang "Development of a masterslave-robot system for farm operations" Computers and Electronics in Agriculture, vol. 44, 2004, pp.1-19.

[14] T. Oksanen, A. Visalam, "Optimal Control of Tractor-Trailer System in Headlines" in Proceedings of the Automation Technology for OffRoad Equipment Conference, Kyoto, Japan, 2004.

[15] D. Bochtis, S. G. Vougioukas, C. Tsatsarelis and Y. Ampatzidis, "Optimal Dynamic Motion Sequence Generation for Multiple Harvesters" in Proceedings of the Automation Technology for OffRoad Equipment Conference, 1-2 September 2006, Bonn, Germany, pp.33-40.

[16] Y. Gao, Q. Zhang "A Comparison of Three Steering Controllers for Off-road Vehicles" in Proceedings of the Automation Technology for Off-Road Equipment Conference, 1-2 September 2006, Bonn, Germany, pp.289-301.

[17] N. Murakami, A. Ito, J.D. Will, M. Steffen, K. Inoue, K. Kita, S. Miyaura "Development of a teleoperation system for agricultural vehicles" Computers and Electronics in Agriculture, vol. 63, 2008, pp. 81-88.

[18] IEC 61508, "Functional safety of electrical/electronic/ programmable electronic safety-related systems", International Electrotechnical Commission, Switzerland. 